\title{
Contraceptive Use Among Nonpregnant and Postpartum Women at Risk for Unintended Pregnancy, and Female High School Students, in the Context of Zika Preparedness — United States, 2011-2013 and 2015
}

\author{
Sheree L. Boulet, DrPH ${ }^{1}$; Denise V. D’Angelo, $\mathrm{MPH}^{1}$; Brian Morrow, MA ${ }^{1}$; Lauren Zapata, $\mathrm{PhD}^{1}$; Erin Berry-Bibee, $\mathrm{MD}^{1}$; Maria Rivera, MPH${ }^{3}$; \\ Sascha Ellington, $\mathrm{MSPH}^{1}$; Lisa Romero, DrPH ${ }^{1}$; Eva Lathrop, $\mathrm{MD}^{4}$; Meghan Frey, MA, MPH${ }^{2}$; Tanya Williams, $\mathrm{MPH}^{1}$; Howard Goldberg, PhD ${ }^{1}$; \\ Lee Warner, $\mathrm{PhD}^{1}$; Leslie Harrison, $\mathrm{MPH}^{1}$; Shanna Cox, MSPH${ }^{1}$; Karen Pazol, $\mathrm{PhD}^{1}$; Wanda Barfield, MD ${ }^{1}$; Denise J. Jamieson, MD ${ }^{1}$; \\ Margaret A. Honein, $\mathrm{PhD}^{2}$; Charlan D. Kroelinger, $\mathrm{PhD}^{1}$
}

\begin{abstract}
On August 2, 2016, this report was posted as an MMWR Early Release on the MMWR website (http://www.cdc.gov/mmwr).
\end{abstract}

Zika virus infection during pregnancy can cause congenital microcephaly and brain abnormalities $(1,2)$. Since 2015, Zika virus has been spreading through much of the World Health Organization's Region of the Americas, including U.S. territories. Zika virus is spread through the bite of Aedes aegypti or Aedes albopictus mosquitoes, by sex with an infected partner, or from a pregnant woman to her fetus during pregnancy. ${ }^{*} \mathrm{CDC}$ estimates that 41 states are in the potential range of Aedes aegypti or Aedes albopictus mosquitoes (3), and on July 29, 2016, the Florida Department of Health identified an area in one neighborhood of Miami where Zika virus infections in multiple persons are being spread by bites of local mosquitoes. These are the first known cases of local mosquito-borne Zika virus transmission in the continental United States. ${ }^{\dagger}$ CDC prevention efforts include mosquito surveillance and control, targeted education about Zika virus and condom use to prevent sexual transmission, and guidance for providers on contraceptive counseling to reduce unintended pregnancy. To estimate the prevalence of contraceptive use among nonpregnant and postpartum women at risk for unintended pregnancy and sexually active female high school students living in the 41 states where mosquito-borne transmission might be possible, CDC used 2011-2013 and 2015 survey data from four state-based surveillance systems: the Behavioral Risk Factor Surveillance System (BRFSS, 2011-2013), which surveys adult women; the Pregnancy Risk Assessment Monitoring System (PRAMS, 2013) and the Maternal and Infant Health Assessment (MIHA, 2013), which surveys women with a recent live birth; and the Youth Risk Behavior Survey (YRBS, 2015), which surveys students in grades 9-12. CDC defines an unintended pregnancy as one that is either unwanted (i.e., the pregnancy occurred when no children, or no more children, were desired) or mistimed (i.e., the pregnancy occurred earlier than desired). The proportion of women at risk for unintended pregnancy who used a highly effective reversible method, known as long-acting reversible contraception (LARC), ranged from 5.5\%

\footnotetext{
${ }^{*}$ http://www.cdc.gov/zika/transmission/.

${ }^{\dagger}$ http://www.cdc.gov/media/releases/2016/p0729-florida-zika-cases.html; http://www.cdc.gov/zika/intheus/florida-update.html.
}

to $18.9 \%$ for BRFSS-surveyed women and $6.9 \%$ to $30.5 \%$ for PRAMS/MIHA-surveyed women. The proportion of women not using any contraception ranged from $12.3 \%$ to $34.3 \%$ (BRFSS) and from $3.5 \%$ to $15.3 \%$ (PRAMS/MIHA). YRBS data indicated that among sexually active female high school students, use of LARC at last intercourse ranged from $1.7 \%$ to $8.4 \%$, and use of no contraception ranged from $7.3 \%$ to $22.8 \%$. In the context of Zika preparedness, the full range of contraceptive methods approved by the Food and Drug Administration (FDA), including LARC, should be readily available and accessible for women who want to avoid or delay pregnancy. Given low rates of LARC use, states can implement strategies to remove barriers to the access and availability of LARC including high device costs, limited provider reimbursement, lack of training for providers serving women and adolescents on insertion and removal of LARC, provider lack of knowledge and misperceptions about LARC, limited availability of youth-friendly services that address adolescent confidentiality concerns, inadequate client-centered counseling, and low consumer awareness of the range of contraceptive methods available.

BRFSS is a cross-sectional, random-digit-dialed, state-based telephone survey that collects data on risk behaviors and preventive health practices among adult respondents living in all 50 states, the District of Columbia, Puerto Rico, Guam, and the U.S. Virgin Islands. ${ }^{\Im}$ Data from 17 states that might be at risk for mosquito-borne transmission of Zika virus (3) and had implemented questions on self-reported contraceptive use as part of the BRFSS Family Planning module in 2011 or as state-added questions in 2012 or 2013 were used to estimate use of contraception among women aged 18-44 years at risk for unintended pregnancy.9 PRAMS is an ongoing state-based and population-based surveillance system designed to monitor selected self-reported maternal behaviors and experiences that occur before, during, and after pregnancy among women who recently delivered a live-born infant. ${ }^{* *}$ Data from 28 PRAMS

\footnotetext{
$\$$ http://www.cdc.gov/brfss/annual_data/2013/pdf/overview_2013.pdf.

9 For BRFSS, women were considered at risk for unintended pregnancy if they were not currently pregnant, were sexually active (not abstinent), and, the last time they had sex, had not had a hysterectomy, did not have a samesex partner, and did not want a pregnancy.

** http://www.cdc.gov/PRAMS/index.htm.
} 
states, reporting in 2013, were used to estimate contraceptive use at the time of the survey (4-6 months postpartum) among women aged $15-44$ years with a recent live birth who were at risk for unintended pregnancy. ${ }^{\dagger \dagger}$ PRAMS sites were included if they might be at risk for mosquito-borne transmission of Zika virus (3) and achieved a weighted response rate of $\geq 55 \%$. $\$ \$$ The 2013 MIHA was used to estimate contraceptive use for postpartum women in California. Using methods comparable to PRAMS, MIHA is an annual, statewide-representative survey of women with a recent live birth.99 YRBSs are conducted by state health and education agencies among representative samples of students in grades 9-12, to monitor health-risk behaviors, including sexual behaviors related to unintended pregnancy and sexually transmitted diseases. ${ }^{* * *}$ Data from 2015 YRBSs conducted in 28 states that might be at risk for mosquito-borne transmission of Zika virus (3) were used to describe contraceptive use among female high school students at last sexual intercourse. ${ }^{\dagger \dagger}$

For all data sources, contraceptive use was classified according to the estimated percentage of users who experience pregnancy during the first year of typical use as highly effective $(<1 \%)$, moderately effective $(6 \%-10 \%)$, and less effective (>10\%) (4). Among women reporting more than one contraceptive method, the most effective method was coded. Highly effective, permanent contraceptive methods included female sterilization, tubal ligation, or partner vasectomy. Highly effective LARC methods included intrauterine devices (IUDs) and contraceptive implants. Moderately effective contraceptive methods included hormone injections, contraceptive pills, transdermal contraceptive patches, and vaginal rings. Less effective methods included diaphragm, condoms (male or female), cervical cap, sponge, withdrawal, spermicide, fertility-based awareness methods, emergency contraception, and "other." Data for the use of permanent contraceptive methods, although included in the denominator for calculating percentages, are not presented

\footnotetext{
${ }^{\dagger \dagger}$ For PRAMS, women were considered at risk for unintended pregnancy if they were not currently pregnant, did not want a pregnancy, were sexually active (not abstinent), and did not report another reason they could not get pregnant (i.e., had a same-sex partner, had a hysterectomy/oopherectomy, or were infertile).

$\$ \$$ PRAMS uses a minimum $60 \%$ response rate for publication. However, based on the critical need to report surveillance data related to Zika virus, PRAMS provided permission to use a lower response rate threshold.

95 MIHA uses the same definition of unintended pregnancy as PRAMS.

*** http://www.cdc.gov/healthyyouth/data/yrbs/methods.htm.

ti† Female high school students were considered currently sexually active if they had sexual intercourse with at least one person during the 3 months before the survey. In 2015, 30.1\% of female high school students nationwide were currently sexually active. http://www.cdc.gov/mmwr/volumes/65/ss/ ss6506a1.htm.
}

because women reporting female sterilization or partner vasectomy do not need ongoing contraceptive services. ${ }^{\$ \$ \$}$

Weighted prevalence estimates and $95 \%$ confidence intervals for contraceptive use were calculated overall and by age group, as appropriate (BRFSS: ages 18-24, 25-34, and 35-44 years; PRAMS/MIHA: ages 15-19, 20-24, 25-34, and 35-44 years) and by race/ethnicity (non-Hispanic white [white], nonHispanic black [black], and Hispanic). For all surveys, nonHispanic other race was included in the denominator, but not presented because of small sample sizes. PRAMS/MIHA data were used to estimate the prevalence of contraceptive use by insurance status (private insurance, Medicaid, and none) 999 ; other insurance was not presented because of small sample sizes. Estimates were excluded when they did not meet the reliability standard established for each surveillance system. ${ }^{* * * *}$

In the 17 states for which BRFSS data were available, use of LARC at last sexual intercourse among women aged 18-44 years at risk for unintended pregnancy ranged from $5.5 \%$ (Arizona) to $18.9 \%$ (Utah) (Table 1). The proportion of women at risk for unintended pregnancy who used no contraception was lowest in Vermont (12.3\%) and highest in Tennessee (34.3\%). For all states, moderately and less effective contraception use was lower among older women (available at https://stacks.cdc.gov/view/cdc/40511). Use of less effective contraception was more common among Hispanic women than among white women (available at https://stacks.cdc.gov/ view/cdc/40511).

PRAMS and MIHA data indicated that the proportion of women aged 15-44 years at risk for unintended pregnancy using LARC during the postpartum period ranged from $6.9 \%$ (New Jersey) to $30.5 \%$ (Utah) (Table 2) and was typically highest among adolescents aged 15-19 years (available at https://stacks.cdc.gov/view/cdc/40512). The proportion of postpartum women at risk for unintended pregnancy who did not use contraception ranged from 3.5\% (Vermont) to $15.3 \%$ (Hawaii). In general, use of LARC and moderately effective contraception was lower in older women (available at https://stacks.cdc.gov/view/cdc/40512). The proportion of women using less effective contraceptive methods tended to be higher among white and Hispanic women than black women (available at https://stacks.cdc.gov/view/cdc/40512). Among

$\$ \$ \$$ In BRFSS, use of highly effective, permanent contraception ranged from $11.7 \%$ to $29.4 \%$; in PRAMS use of highly effective, permanent contraception ranged from $7.5 \%$ to $18.8 \%$. YRBSs do not collect information on highly effective, permanent methods of contraception.

999 Insurance status was reported at the time of survey, between 4 and 6 months postpartum.

**** BRFSS data were excluded if unweighted denominators had $<50$ respondents or a relative standard error $>30 \%$. PRAMS and MIHA data were suppressed if unweighted denominators had $<30$ respondents; estimates based on $<60$ respondents were flagged and should be interpreted with caution. YRBS data were suppressed if there were $<100$ respondents. 
TABLE 1. Use of contraception* at last sexual intercourse among women aged 18-44 years at risk for unintended pregnancy, ${ }^{\dagger}$ by selected states where mosquito-borne Zika virus transmission might be possible and data were available -10 states ${ }^{\S}$ with state-added questions on reproductive health, Behavioral Risk Factor Surveillance System (BRFSS) survey, 2013, two states " with state-added questions on reproductive health (BRFSS, 2012) and five states** with state-added questions on reproductive health (BRFSS, 2011)

\begin{tabular}{|c|c|c|c|c|c|c|}
\hline \multirow[b]{2}{*}{ State } & \multirow[b]{2}{*}{ Unweighted no. } & \multirow{2}{*}{$\begin{array}{c}\text { Weighted } \\
\text { no. }\end{array}$} & \multirow{2}{*}{$\begin{array}{c}\begin{array}{c}\text { Highly effective, } \\
\text { reversible (LARC) }\end{array} \\
\%(95 \% \mathrm{Cl})\end{array}$} & \multirow{2}{*}{$\frac{\text { Moderately effective }{ }^{\S \S}}{\%(95 \% \mathrm{Cl})}$} & \multirow{2}{*}{$\frac{\text { Less effective }}{\% \text { ๆी }}$} & \multirow{2}{*}{$\frac{\text { None }}{\%(95 \% \mathrm{Cl})}$} \\
\hline & & & & & & \\
\hline Arizona & 307 & 538,319 & $5.5(3.4-8.9)$ & $17.9(12.5-25.1)$ & $23.6(17.3-31.2)$ & $32.0(25.1-39.9)$ \\
\hline Colorado & 587 & 599,782 & $15.4(12.2-19.3)$ & $27.7(23.6-32.2)$ & $17.1(13.7-21.1)$ & $15.8(12.6-19.6)$ \\
\hline Connecticut & 547 & 440,679 & $9.6(6.2-14.6)$ & $25.4(19.5-32.4)$ & $23.2(18.0-29.2)$ & $26.1(20.5-32.6)$ \\
\hline Florida & 762 & $1,334,658$ & $6.8(4.6-10.0)$ & $16.6(13.2-20.6)$ & $25.0(20.4-30.1)$ & $27.5(22.9-32.6)$ \\
\hline Kentucky & 884 & 523,533 & $6.9(5.1-9.3)$ & $24.2(20.5-28.4)$ & $18.0(14.8-21.7)$ & $22.8(19.1-27.0)$ \\
\hline Massachusetts & 753 & 866,004 & $14.0(10.6-18.1)$ & $23.9(19.4-29.2)$ & $20.2(16.3-24.8)$ & $30.2(24.6-36.5)$ \\
\hline Mississippi & 461 & 325,091 & $6.5(4.2-9.8)$ & $21.4(17.2-26.3)$ & $24.9(20.2-30.4)$ & $18.7(14.8-23.4)$ \\
\hline Missouri & 418 & 502,152 & $7.6(5.2-11.1)$ & $17.5(13.3-22.7)$ & $23.4(18.0-29.8)$ & $25.2(19.8-31.4)$ \\
\hline New York & 2,728 & $2,135,002$ & $11.8(7.3-18.6)$ & $26.0(20.3-32.7)$ & $26.1(20.8-32.2)$ & $22.2(17.6-27.5)$ \\
\hline North Carolina & 676 & 691,264 & $8.3(5.9-11.6)$ & $22.7(18.5-27.4)$ & $24.3(20.0-29.2)$ & $24.2(20.2-28.7)$ \\
\hline Ohio & 658 & $1,386,428$ & $10.0(7.3-13.5)$ & $21.6(17.6-26.2)$ & $18.7(14.5-23.8)$ & $29.4(24.9-34.3)$ \\
\hline Pennsylvania & 1,821 & $1,336,494$ & $7.6(6.1-9.4)$ & $22.6(20.1-25.3)$ & 24.7 (21.8-27.9) & $24.1(21.0-27.3)$ \\
\hline South Carolina & 1,356 & 543,085 & $6.6(4.9-9.0)$ & $26.6(23.3-30.2)$ & $21.0(18.3-23.9)$ & $22.7(19.7-26.0)$ \\
\hline Tennessee & 557 & 592,990 & $6.5(3.9-10.6)$ & $13.8(9.2-20.1)$ & $16.0(11.1-22.6)$ & $34.3(27.4-42.0)$ \\
\hline Texas & 347 & $3,061,291$ & $10.1(5.5-17.7)$ & $23.1(17.6-29.8)$ & $17.3(12.5-23.5)$ & $26.1(20.2-33.1)$ \\
\hline Utah & 656 & 256,840 & $18.9(15.3-23.0)$ & $20.7(17.2-24.6)$ & $21.7(18.0-25.8)$ & $18.9(15.5-23.0)$ \\
\hline Vermont & 605 & 70,062 & $13.8(11.0-17.3)$ & $30.2(25.6-35.2)$ & $20.8(17.0-25.2)$ & $12.3(9.4-16.0)$ \\
\hline
\end{tabular}

Abbreviations: $\mathrm{LARC}=$ long-acting, reversible contraception; $\mathrm{Cl}=$ confidence interval.

* Women using permanent contraception were included in the denominator for all estimates.

† Women were considered at risk for unintended pregnancy if they were not currently pregnant, were sexually active (not abstinent), and, the last time they had sex,

had not had a hysterectomy, did not have a same-sex partner, and did not want a pregnancy.

$\S$ Arizona, Connecticut, Kentucky, Massachusetts, Mississippi, New York (data collected April 2013-March 2014), Ohio, Texas, Utah, and Vermont.

" Pennsylvania and Colorado.

** Florida, Missouri, North Carolina, South Carolina, and Tennessee.

t+ Highly effective, reversible contraceptive methods or LARC include intrauterine devices and implants.

$\S \S$ Moderately effective contraceptive methods include hormone injections, contraceptive pills, transdermal contraceptive patch, and vaginal ring.

ๆศ Less effective contraceptive methods include diaphragm, condoms (male or female), cervical cap, sponge, withdrawal, spermicide, fertility-based awareness methods, emergency contraception, and "other." Respondents answering "other" were given the opportunity to write in a response, which was evaluated and reclassified into existing contraceptive method options as appropriate. For Connecticut, Kentucky, Massachusetts, Mississippi, Ohio, Texas and Utah, text responses for "other" contraception were evaluated and reclassified into appropriate categories when possible. The text field was not available for other states.

women with no insurance, use of LARC ranged from 5.3\% (New Jersey) to $34.2 \%$ (Utah) (available at https://stacks.cdc. gov/view/cdc/40513).

YRBS data indicated that among currently sexually active female high school students in 28 states, LARC use ranged from $<2 \%$ (North Carolina and Pennsylvania) to $8.4 \%$ (Vermont) (Table 3). Use of less effective contraceptive methods ranged from $36.3 \%$ (Vermont) to $59.9 \%$ (Florida); the proportion of sexually active female high school students not using any contraception was lowest in Vermont $(7.3 \%)$ and highest in Arkansas (22.8\%). Limited data were available to describe sexually active female high school students using contraception by method effectiveness and race/ethnicity (available at https:// stacks.cdc.gov/view/cdc/40514).

\section{Discussion}

During 2011-2013 and 2015, nonpregnant and postpartum women at risk for unintended pregnancy, and sexually active female high school students in states that might be at risk for mosquito-borne transmission of Zika virus, used moderately effective and less effective contraceptive methods most frequently; use of no contraception varied among states. LARC was used by fewer than one fourth of nonpregnant women, approximately one third of women who recently delivered a live birth, and fewer than one tenth of sexually active female high school students. LARC use also varied by state, age group, race/ethnicity, and insurance status. Increasing accessibility of contraceptive services, including LARC, can reduce unintended pregnancy, including the number of pregnancies affected by Zika virus infection among women who are returning, or whose partners are returning, from areas with ongoing Zika virus transmission (5).

Despite the availability of a wide range of FDA-approved contraceptives, unintended pregnancy remains common in the United States; the most recent estimates indicate that $45 \%$ of all pregnancies are unintended $(6)$, with variation across states ( 7$)$ and by age group, income, education, and race/ethnicity (G). LARC methods are highly effective, reversible methods for reducing unintended pregnancy, do not depend on user compliance, and are medically appropriate for most female 
Morbidity and Mortality Weekly Report

TABLE 2. Use of postpartum contraception* among women aged 15-44 years who recently had a live birth and were at risk for unintended pregnancy, ${ }^{\dagger}$ by selected states where mosquito-borne Zika virus transmission might be possible and data were available - Pregnancy Risk Assessment Monitoring System and Maternal Infant and Health Assessment, $\$ 2013$

\begin{tabular}{|c|c|c|c|c|c|c|}
\hline & & & $\begin{array}{l}\text { Highly effective, } \\
\text { reversible (LARC) }\end{array}$ & Moderately effective ${ }^{* *}$ & Less effective $^{\dagger \dagger}$ & None \\
\hline State & Unweighted no. & Weighted no. & $\%(95 \% \mathrm{Cl})$ & $\%(95 \% \mathrm{Cl})$ & $\%(95 \% \mathrm{Cl})$ & $\%(95 \% \mathrm{CI})$ \\
\hline Arkansas & 872 & 29,978 & $13.1(9.9-17.2)$ & $35.5(30.7-40.7)$ & $26.8(22.4-31.7)$ & $5.8(3.9-8.6)$ \\
\hline California & 6,037 & 414,243 & $15.4(13.7-17.1)$ & $29.2(26.9-31.5)$ & $37.6(35.0-40.2)$ & $6.2(5.0-7.4)$ \\
\hline Colorado & 1,466 & 56,393 & $24.7(21.8-28.0)$ & $26.0(22.9-29.2)$ & $30.3(27.1-33.7)$ & $5.6(4.2-7.4)$ \\
\hline Connecticut & 1,021 & 29,364 & $20.9(17.7-24.5)$ & $28.5(25.0-32.2)$ & $35.2(31.0-39.7)$ & $7.9(5.7-10.8)$ \\
\hline Florida & 1,103 & 179,043 & $14.7(12.4-17.4)$ & $32.8(29.5-36.3)$ & $28.9(25.8-32.2)$ & $8.0(6.2-10.1)$ \\
\hline Georgia & 665 & 58,334 & $18.5(14.2-23.6)$ & $39.0(33.3-45.0)$ & $15.6(11.7-20.5)$ & $8.7(5.9-12.5)$ \\
\hline Hawaii & 1,216 & 15,075 & $17.8(15.0-21.0)$ & $33.4(30.0-37.1)$ & $24.6(21.5-28.0)$ & $15.3(12.6-18.4)$ \\
\hline Illinois & 1,156 & 123,604 & $16.8(14.6-19.4)$ & $34.9(31.9-38.0)$ & $30.3(27.5-33.2)$ & $7.5(6.0-9.4)$ \\
\hline lowa & 1,012 & 32,421 & $18.9(15.7-22.7)$ & $37.3(33.0-41.8)$ & $24.3(20.7-28.4)$ & $4.6(3.1-7.0)$ \\
\hline Louisiana & 1,316 & 51,925 & $10.8(8.7-13.4)$ & 42.7 (39.1-46.4) & $23.6(20.6-27.0)$ & $8.1(6.3-10.5)$ \\
\hline Maine & 809 & 10,519 & $25.5(22.1-29.1)$ & $28.0(24.6-31.7)$ & $27.2(23.8-30.8)$ & 6.9 (5.2-9.3) \\
\hline Maryland & 1,047 & 52,718 & $12.7(10.5-15.4)$ & $34.5(31.2-38.0)$ & $32.1(28.9-35.5)$ & $10.7(8.6-13.1)$ \\
\hline Massachusetts & 1,203 & 57,967 & $20.8(17.9-24.0)$ & $33.2(29.6-37.0)$ & $32.0(28.5-35.7)$ & $5.0(3.6-6.9)$ \\
\hline Minnesota & 1,140 & 56,367 & $20.0(17.4-22.8)$ & $31.3(28.3-34.4)$ & $31.7(28.7-34.8)$ & $7.1(5.6-9.1)$ \\
\hline Missouri & 1,030 & 62,628 & $19.2(16.6-22.0)$ & $31.5(28.4-34.8)$ & $29.0(26.0-32.2)$ & $5.9(4.4-7.7)$ \\
\hline Nebraska & 1,352 & 21,887 & $16.4(14.2-18.9)$ & $32.6(29.7-35.7)$ & $30.7(27.8-33.6)$ & $8.3(6.7-10.2)$ \\
\hline New Hampshire & 550 & 10,793 & 23.8 (19.7-28.5) & $29.0(24.7-33.8)$ & $27.8(23.5-32.4)$ & $5.8(3.9-8.6)$ \\
\hline New Jersey & 742 & 51,983 & $6.9(5.1-9.3)$ & $32.8(29.2-36.7)$ & $35.4(31.7-39.3)$ & $11.6(9.3-14.4)$ \\
\hline New Mexico & 1,435 & 21,521 & $26.7(24.3-29.3)$ & $33.4(30.8-36.0)$ & $20.8(18.6-23.2)$ & $5.9(4.7-7.3)$ \\
\hline New York§§ & 976 & 87,301 & $13.6(10.8-17.1)$ & $32.6(28.4-37.2)$ & $32.1(28.0-36.5)$ & $9.4(6.9-12.5)$ \\
\hline Ohio & 1,237 & 113,373 & $14.4(12.0-17.2)$ & $34.7(31.3-38.3)$ & $26.3(23.3-29.6)$ & $8.8(7.0-11.1)$ \\
\hline Oklahoma & 1,598 & 44,927 & $19.3(16.1-22.9)$ & $35.3(31.3-39.5)$ & 22.7 (19.4-26.4) & $6.2(4.5-8.4)$ \\
\hline Pennsylvania & 874 & 110,078 & $12.5(10.1-15.2)$ & $33.8(30.3-37.5)$ & $34.3(30.8-37.9)$ & $8.2(6.4-10.5)$ \\
\hline Rhode Island & 1,002 & 8,604 & $25.4(22.6-28.5)$ & $31.8(28.6-35.0)$ & $24.3(21.5-27.4)$ & $5.5(4.1-7.2)$ \\
\hline Tennessee & 632 & 65,647 & $13.0(10.0-16.8)$ & $41.6(36.8-46.6)$ & $21.3(17.5-25.6)$ & $6.2(4.1-9.1)$ \\
\hline Texas & 1,046 & 322,651 & $14.7(12.2-17.7)$ & $32.4(29.0-36.0)$ & $33.1(29.6-36.8)$ & $5.4(3.9-7.4)$ \\
\hline Utah & 1,250 & 44,789 & $30.5(27.4-33.7)$ & $25.8(22.9-28.9)$ & $29.9(26.8-33.2)$ & $5.7(4.3-7.5)$ \\
\hline Vermont & 832 & 5,040 & $23.6(20.7-26.7)$ & $30.2(27.1-33.6)$ & $30.8(27.7-34.1)$ & $3.5(2.4-5.0)$ \\
\hline Wisconsin & 1,277 & 53,629 & $16.6(13.6-20.2)$ & 34.7 (30.7-38.9) & $29.6(25.8-33.8)$ & $6.2(4.3-8.9)$ \\
\hline
\end{tabular}

Abbreviations: $\mathrm{LARC}=$ long-acting, reversible contraception; $\mathrm{Cl}=$ confidence interval.

* Women using permanent contraception were included in the denominator for all estimates.

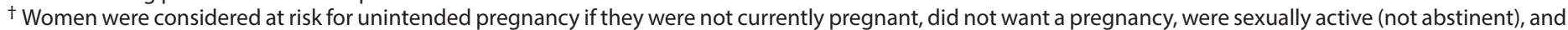
did not report another reason they could not get pregnant (i.e., had a same-sex partner, had a hysterectomy/oopherectomy, or were infertile).

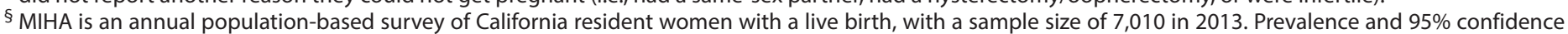
intervals are weighted to represent all women with a live birth in California in 2013.

" Highly effective, reversible contraceptive methods or LARC include intrauterine devices and implants.

** Moderately effective contraceptive methods include hormone injections, contraceptive pills, transdermal contraceptive patch, and vaginal ring.

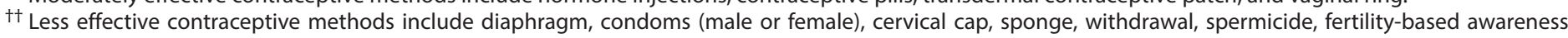

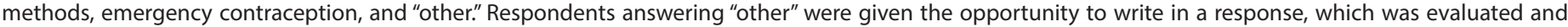
reclassified into existing contraceptive method options as appropriate.

$\S \S$ Does not include New York City.

adolescents and adult women $(4,8)$. Nationally, although use of LARC methods nearly doubled in recent years (9), use remains lower than that of other reversible contraceptives such as oral contraceptive pills and condoms (9), and considerable barriers to access and contraceptive method availability remain (10).

The most recent estimates for the United States suggest that lower income women had rates of unintended pregnancy up to five times higher than women with higher incomes (G). During 2000-2010, the need for publicly funded contraceptive services increased $17 \%$ (11). ${ }^{\dagger \dagger \dagger}$ Although publicly funded providers

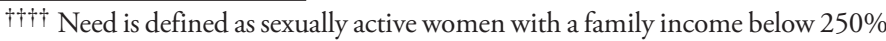
of the federal poverty level and all women younger than age 20 years, who are able to conceive and were not intentionally trying to get pregnant.
}

met approximately $42 \%$ of contraceptive need in 2013 , unmet need varied by state, suggesting gaps in access to subsidized contraceptive care (11). Among low income women with Medicaid insurance, recent guidance emphasizes provision of contraceptive services without cost-sharing. ${ }^{\$ \$}$ Also, whereas women with private insurance coverage reported decreased out-of-pocket costs for LARC following the 2012 Affordable Care Act requirement for most private health plans to cover contraceptive services, $13 \%$ of women continued to cost-share (12), further highlighting differences in access and availability (13). Although federal regulations for publicly funded coverage enable minors to obtain contraceptive care without parental

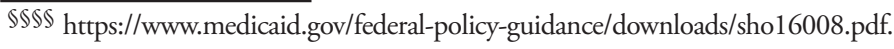


Morbidity and Mortality Weekly Report

TABLE 3. Use of contraception at last sexual intercourse among female students in grades 9-12 who were currently sexually active, ${ }^{*}$ by selected states where mosquito-borne Zika virus transmission might be possible and data were available — Youth Risk Behavior Survey, 2015

\begin{tabular}{|c|c|c|c|c|c|}
\hline & & $\begin{array}{l}\text { Highly effective, reversible } \\
{\text { (LARC) })^{\dagger}}\end{array}$ & Moderately effective ${ }^{\S}$ & Less effective ${ }^{\text {I }}$ & None \\
\hline State & Unweighted no. & $\%(95 \% \mathrm{Cl})$ & $\%(95 \% \mathrm{Cl})$ & $\%(95 \% \mathrm{Cl})$ & $\%(95 \% \mathrm{Cl})$ \\
\hline Alabama & 204 & $2.9(1.2-6.5)$ & $36.2(27.6-45.7)$ & $39.7(34.1-45.6)$ & $18.5(12.6-26.5)$ \\
\hline Arizona & 319 & $5.4(2.0-13.8)$ & $23.5(14.8-35.3)$ & $54.3(48.1-60.4)$ & $15.3(9.4-23.9)$ \\
\hline Arkansas & 378 & $2.4(0.9-6.2)$ & $30.1(22.3-39.1)$ & $43.2(32.8-54.2)$ & $22.8(18.2-28.3)$ \\
\hline California & 199 & $5.3(2.4-11.3)$ & $19.9(13.0-29.2)$ & $55.3(48.1-62.2)$ & $12.4(8.9-16.9)$ \\
\hline Connecticut & 245 & $3.8(2.0-7.4)$ & $32.0(24.6-40.6)$ & $48.9(40.3-57.6)$ & $12.3(7.8-18.7)$ \\
\hline Delaware & 399 & $4.3(2.4-7.5)$ & $30.8(25.5-36.6)$ & $48.6(41.6-55.7)$ & $14.1(8.0-23.6)$ \\
\hline Florida & 669 & $2.0(1.1-3.5)$ & $19.7(16.1-23.8)$ & $59.9(54.9-64.7)$ & $16.5(14.0-19.3)$ \\
\hline Hawaii & 687 & $6.1(3.1-11.5)$ & $25.3(21.3-29.8)$ & $54.8(48.4-61.1)$ & $11.4(7.8-16.4)$ \\
\hline Illinois & 363 & $4.8(2.7-8.6)$ & $36.7(27.0-47.6)$ & $46.2(38.2-54.4)$ & $11.8(8.0-16.9)$ \\
\hline Indiana & 237 & $5.0(2.4-10.0)$ & $32.1(22.3-43.9)$ & $44.0(36.2-52.0)$ & $16.3(10.7-24.0)$ \\
\hline Kentucky & 325 & $7.8(3.9-15.1)$ & $36.6(27.6-46.6)$ & $37.0(28.0-47.0)$ & $17.5(13.9-22.0)$ \\
\hline Maine & 1196 & $6.4(4.1-9.9)$ & $43.6(41.2-46.1)$ & $40.5(36.9-44.2)$ & $8.6(6.9-10.7)$ \\
\hline Maryland & 5,572 & $2.6(2.1-3.2)$ & $27.8(26.4-29.3)$ & $52.8(51.2-54.3)$ & $15.3(14.0-16.7)$ \\
\hline Massachusetts & 388 & $6.0(3.6-10.1)$ & $36.2(30.3-42.6)$ & $48.1(40.9-55.3)$ & $9.2(6.7-12.6)$ \\
\hline Mississippi & 246 & $4.8(2.6-8.8)$ & $30.8(23.5-39.3)$ & $49.7(42.8-56.6)$ & $14.0(8.5-22.2)$ \\
\hline Missouri & 172 & $4.9(2.5-9.2)$ & $35.8(27.1-45.6)$ & $48.1(41.8-54.3)$ & 10.5 (5.6-19.0) \\
\hline Nebraska & 173 & $5.0(2.3-10.2)$ & $28.9(21.5-37.5)$ & $45.6(36.2-55.4)$ & $19.0(11.9-29.0)$ \\
\hline Nevada & 183 & $2.7(0.6-11.1)$ & $28.8(18.3-42.3)$ & $54.8(40.2-68.6)$ & $13.8(9.7-19.1)$ \\
\hline New Hampshire & 2,239 & $6.6(5.4-8.0)$ & $43.6(40.1-47.2)$ & $41.6(38.0-45.3)$ & $7.4(6.0-9.0)$ \\
\hline New Mexico & 968 & $7.8(6.0-10.2)$ & $25.7(22.3-29.5)$ & $45.9(41.6-50.3)$ & $17.5(14.2-21.2)$ \\
\hline New York & 930 & $4.7(2.1-10.5)$ & $30.4(24.8-36.5)$ & $49.6(41.8-57.5)$ & $13.6(10.9-16.9)$ \\
\hline North Carolina & 774 & $1.9(1.0-3.5)$ & $27.0(21.3-33.7)$ & $53.9(45.8-61.8)$ & $15.4(10.4-22.4)$ \\
\hline Oklahoma & 197 & $3.8(1.4-9.6)$ & $23.6(17.5-31.0)$ & $54.2(45.9-62.3)$ & $15.4(9.8-23.3)$ \\
\hline Pennsylvania & 340 & $1.7(0.8-3.7)$ & $28.9(23.5-35.1)$ & $56.8(51.1-62.3)$ & $12.2(8.7-16.8)$ \\
\hline Rhode Island & 413 & $3.7(1.6-8.1)$ & $31.1(26.0-36.7)$ & $49.7(43.0-56.3)$ & $13.4(9.3-19.1)$ \\
\hline South Carolina & 156 & $6.5(1.7-22.3)$ & $32.7(25.0-41.4)$ & $44.6(38.5-50.8)$ & $14.2(10.5-18.9)$ \\
\hline Vermont & 3,028 & $8.4(7.5-9.5)$ & $47.0(45.2-48.8)$ & $36.3(34.6-38.0)$ & $7.3(6.4-8.3)$ \\
\hline West Virginia & 278 & $3.1(1.3-7.0)$ & $41.7(34.2-49.6)$ & $41.0(33.4-49.2)$ & $11.3(8.5-15.0)$ \\
\hline
\end{tabular}

Abbreviations: $\mathrm{LARC}=$ long-acting, reversible contraception; $\mathrm{Cl}=$ confidence interval.

* Had sexual intercourse with at least one person during the 3 months before the survey.

† Highly effective, reversible contraceptive methods or LARC include intrauterine devices (e.g., Mirena or ParaGard) and implants (e.g., Implanon or Nexplanon).

$\$$ Moderately effective contraceptive methods include oral contraceptive pills or a hormone injection (e.g., Depo-Provera), a transdermal patch (e.g., OrthoEvra), or a vaginal birth control ring (e.g., NuvaRing).

$\checkmark$ Less effective contraceptive methods include condoms to prevent pregnancy, withdrawal, or some other method.

consent, private insurers often follow state laws, which vary by jurisdiction, potentially limiting access (14). To improve access and availability to the full range of contraception, a number of state-level and jurisdictional-level strategies exist and could be adopted by state and local agencies (Box).

The findings in this report are subject to at least five limitations. First, information on contraceptive use was self-reported and might be subject to recall or social desirability bias, and response rates varied by state and surveillance system.9999 Second, consistent and correct use of contraception affects effectiveness rates, and this was not measured. Third, population estimates are generalizable only to specific populations for which data are collected; for example, estimates among

\footnotetext{
9999 BRFSS response rates vary by state (https://www.cdc.gov/brfss/annual_ data/2013/pdf/2013_dqr.pdf). PRAMS/MIHA response rates vary by state, but must meet the minimum $55 \%$ response threshold to be included; however, the typical minimum response threshold for PRAMS/MIHA is 65\% (http:// www.cdc.gov/prams/methodology.htm). YRBS response rates vary by state (http://www.cdc.gov/mmwr/preview/mmwrhtml/rr6201a1.htm).
}

sexually active female high school students are not generalizable to adolescents who do not attend school. Fourth, the current contraceptive use profile in states might have changed since the data were collected. Finally, only 39 of the 41 states had data from at least one surveillance system, highlighting the need for ongoing collection of state-level data on contraceptive use (3).*****

State-level strategies for increasing access to the full range of FDA-approved contraceptive methods and related services can reduce unintended pregnancies among women, including women who might be exposed to Zika virus. CDC supports states in 1) implementing vector control strategies;

\footnotetext{
***** The 41 states in the potential range of Zika-carrying mosquitoes are as follows: Alabama, Arizona, Arkansas, California, Colorado, Connecticut, Delaware, Florida, Georgia, Hawaii, Illinois, Indiana, Iowa, Kansas, Kentucky, Louisiana, Maine, Maryland, Massachusetts, Minnesota, Mississippi, Missouri, Nebraska, Nevada, New Hampshire, New Jersey, New Mexico, New York, North Carolina, Ohio, Oklahoma, Pennsylvania, Rhode Island, South Carolina, Tennessee, Texas, Utah, Vermont, Virginia, West Virginia, and Wisconsin.
} 
BOX. State and jurisdictional-level strategies for increasing access and availability of long-acting reversible contraception (LARC) by state and local agencies, health systems, and providers

Facilitate partnership among private and public insurers, device manufacturers, and state agencies

- Improve acquisition management

- Streamline service provision

- Increase efficiency in product purchase

- Reduce per capita costs

Reimburse providers for the full range of contraceptive services

- Screen for pregnancy intention

- Provide client-centered contraception counseling

- Fund full cost of device insertion, removal, and replacement

- Compensate for device reinsertion of LARC and follow-up

Remove logistic and administrative barriers for contraceptive services and supplies*

- Eliminate policies requiring pre-approval

- Decrease step therapy restriction or required use of generic drugs before brand-name medication

- Stock highly effective contraceptive devices in all hospitals and clinics

Train health care providers on current insertion and removal techniques for LARC

- Support use of CDC's evidence-based contraceptive guidance ${ }^{\dagger}$

- Provide quality family planning services $\$$

- Increase awareness on use of LARC for most clients of all ages

Support youth-friendly reproductive health services

- Educate health care providers on confidentiality concerns of female adolescents/minors

- Withhold automated distribution of explanation of benefits to the primary payer

- Offer extended and weekend hours

- Provide teen-focused, culturally appropriate materials

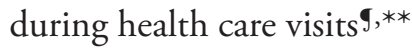

\section{Engage smaller or rural facilities including}

community health care centers ${ }^{\dagger \dagger}$

- Ensure adequate provider training and supply of LARC

- Partner with larger facilities to implement contraceptive services
BOX. (Continued) State and jurisdictional-level strategies for increasing access and availability of long-acting reversible contraception (LARC) by state and local agencies, health systems, and providers

Assess client satisfaction with service provision ${ }^{\$ \S}$ and increase consumer awareness

- Implement public/private campaigns

- Provide comprehensive sexual health education in secondary schools

* Auerbach J. The 3 buckets of prevention. J Public Health Manag Pract 2016;22:215-8.

$\dagger$ Curtis KM, Jatloui TC, Tepper NK. U.S. selected practice recommendations for contraceptive use, 2016. MMWR Recomm Rep 2016;65(No. RR-4).

$\$$ Gavin L, Moskosky S, Carter M, et al. Providing quality family planning services: recommendations of CDC and the U.S. Office of Population Affairs. MMWR Recomm Rep 2014;63(No. RR-4).

9 Hathaway M, Torres L, Vollett-Krech J, Wohltjen H. Increasing LARC utilization: any woman, any place, any time. Clin Obstet Gynecol 2014;57:718-30.

** CDC. Communitywide teen pregnancy prevention initiatives. Atlanta, GA: US Department of Health and Human Services, CDC; 2015. http:// www.cdc.gov/teenpregnancy/prevent-teen-pregnancy!

t† Goldberg DG, Wood SF, Johnson K, et al. The organization and delivery of family planning services in community health centers. Womens Health Issues 2015;25:202-8.

$\$ \$$ Berwick DM, Nolan TW, Whittington J. The triple aim: care, health, and cost. Health Aff (Millwood) 2008;27:759-69.

2) identifying, diagnosing, and clinically managing infection and exposure among pregnant women; and 3) increasing information about effective contraception to avoid unintended pregnancy (15). Prevention efforts for all women and men of reproductive age include targeted education about Zika virus and its transmission, condom use to avoid sexual transmission to pregnant women, and contraceptive counseling for women who want to delay or avoid pregnancy (15). Because contraception is the primary means to prevent unintended pregnancy for women at risk for Zika virus infection, sexually active nonpregnant women of reproductive age and their sex partners need to have access to all approved contraceptive methods, and these methods need to be readily available and accessible.

\section{Acknowledgments}

Behavioral Risk Factor Surveillance System (BRFSS) state collaborators: Rachel (Eddington) Allred, Utah Department of Health; Amy Anderson, Colorado Department of Public Health and Environment; Diane Aye, Connecticut Department of Public Health; Judy Bass, Arizona Department of Health Services; Dana Bernson, Massachusetts Department of Public Health; Carolyn Cass, Pennsylvania Department of Health; James Cassell, North Carolina Department of Health and Human Services; Charlene Collier, Mississippi State Health department; Navina Forsyth, Utah Department of Health; Jill Garratt, Ohio Department of Health; 


\section{Summary}

What is already known on this topic?

Zika virus is transmitted through the bite of an Aedes species mosquito, sex with an infected partner, or from a pregnant woman to her fetus. Zika virus infection during pregnancy is a cause of congenital microcephaly and other severe fetal brain defects. It has also been associated with eye defects, hearing loss, and impaired growth. Nearly half of all pregnancies in the United States are unintended. Among nonpermanent contraceptive methods, long-acting reversible contraception (LARC) is the most effective contraceptive option for preventing unintended pregnancy.

What is added by this report?

State-based estimates of contraception use are provided for nonpregnant and postpartum women at risk for unintended pregnancy and sexually active female high school students. Among these populations, use of moderate and less effective contraception was most common; use of no contraceptive method and use of LARC varied by state, age group, and race/ethnicity.

What are the implications for public health practice?

State and local strategies are needed to increase access to contraceptive methods and related services, reduce the risk for unintended pregnancy, and minimize the number of pregnancies affected by Zika infection. Potentially effective strategies include addressing policies on high device costs and provider reimbursement, comprehensive provider training on insertion and removal of LARC, provision of youth-friendly services, support to resource-challenged jurisdictions, client-centered counseling and assessment of patient satisfaction, and increased consumer awareness of the full range of contraceptive methods to delay or avoid pregnancy.

Alison Grace Bui, Colorado Department of Public Health and Environment; Jessie Hammond, Vermont Department of Health; Junwei Jiang, Florida Department of Health; Sarojini Kanotra, Kentucky Department for Public Health; Chelsea Lynes, South Carolina Department of Health and Environmental Control; Ron McAnally, Mississippi State Health Department; Maria McKenna, Massachusetts Department of Public Health; Arthur Pashi, Missouri Department of Health and Senior Services; Alden Small, Pennsylvania Department of Health; Mycroft Sowizral, New York State Department of Health; Carol Stone, Connecticut Department of Public Health; Rebecca Wood, Texas Department of State Health Services; Ransom Wyse, Tennessee Department of Health; Shumei Yun, Missouri Department of Health and Senior Services; Lei Zhang, Mississippi State Health Department. CDC collaborators: Susan Hocevar Adkins, Division of Adolescent and School Health, National Center for HIV/AIDS, Viral Hepatitis, STD, and TB Prevention (NCHHSTP); Danielle Barradas, Division of Reproductive Health, National Center for Chronic Disease Prevention and Health Promotion (NCCDPHP); Lisa Barrios, Division of Adolescent and School Health, NCHHSTP; Denise Bradford, Division of Adolescent and School Health, NCHHSTP; Kim Burley, Division of Reproductive Health, NCCDPHP; Deborah
Dee, Division of Reproductive Health, NCCDPHP; Heather Clayton, Division of Adolescent and School Health, NCHHSTP; Sara Crawford, Division of Reproductive Health, NCCDPHP; Kate Curtis, Division of Reproductive Health, NCCDPHP; William Garvin, Division of Population Health, NCCDPHP; Violanda Grigorescu, Division of Health Informatics and Surveillance, Center for Surveillance, Epidemiology and Laboratory Services; Laura Kann, Division of Adolescent and School Health, NCHHSTP; Sachiko Kuwabara, Division of Emergency Operations, Office of Public Health Preparedness and Response; Tim McManus, Division of Adolescent and School Health, NCHHSTP; Emily Olsen, Division of Adolescent and School Health, NCHHSTP; Ghasi Phillips-Bell, Division of Reproductive Health, NCCDPHP; Ruben Smith, Division of Reproductive Health, NCCDPHP; Tenecia Smith, Office on Smoking and Health, NCCDPHP; Gary Stuart, Division of Reproductive Health, NCCDPHP; Machell Town, Division of Population Health, NCCDPHP; Guixiang Zhao, Division of Population Health, NCCDPHP; CDC Pregnancy Risk Assessment Monitoring System, Division of Reproductive Health. Maternal and Infant Health Assessment (MIHA) collaborators: Michael Curtis, California Department of Public Health; Melanie Dove, California Department of Public Health; Dawnte Early, California Department of Public Health; Katherine Heck, University of California, San Francisco; Kristen Marchi, University of California, San Francisco; Christine Rinki, California Department of Public Health; Monisha Shah, University of California, San Francisco. Pregnancy Risk Assessment Monitoring System (PRAMS) working group: Izza Afgan, Alabama Department of Public Health; Melissa Baker, West Virginia Department of Health and Human Resources; Claudia W. Bingham, Oregon Health Authority; Peggy Brozicevic, Vermont Department of Health; Brenda Coufal, Nebraska Department of Health \& Human Services; Oralia Flores, New Mexico Department of Health; Connie Geidenberger, Ohio Department of Health; Jihae Goo, Hawaii Department of Health; Mira Grice Sheff, Minnesota Department of Health; Tanya Guthrie, Texas Department of State Health Services; Peterson Haak, Michigan Department of Health and Human Services; Christopher Hill, Virginia Department of Health; Christopher Huard, Wisconsin Department of Health Services; Brenda Hughes, Mississippi State Health Department; Kathleen Jones-Vessey, North Carolina Department of Health and Human Services; Patricia Kloppenburg, Illinois Department of Public Health; Lakota Kruse, New Jersey Department of Health \& Senior Services; David J. Laflamme, New Hampshire Department of Health and Human Services; Ramona Lainhart, Tennessee Department of Health; Alicia Lincoln, Oklahoma Department of Health; Linda Lohdefinck, Washington State Department of Health; Emily Lu, Massachusetts Department of Public Health; Laurie Kettinger, Maryland Department of Health and Mental Hygiene; Sarah Mauch, Iowa Department of Public Health; David McBride, Missouri Department of Health and Senior Services; Mary McGehee, Arkansas Department of Health; Jennifer Morin, Connecticut Department of Public Health; Candace Mulready-Ward, New York City Department of Health and Mental Hygiene; Tony Norwood, Pennsylvania Department of Health; Megan O'Connor, Louisiana Department of 
Health and Hospitals; Tom Patenaude, Maine Department of Health and Human Services; Kathy Perham-Hester, Alaska Department of Health and Social Services; Anne Radigan, New York State Department of Health; Alyson Shupe, Colorado Department of Public Health and Environment; Alisa Simon, Florida Department of Health; Mike Smith, South Carolina Department of Health and Environmental Control; Amy Spieker, Wyoming Department of Health; Nicole Stone, Utah Department of Health; Karine Tolentino Monteiro, Rhode Island Department of Health; George Yocher, Delaware Department of Health and Social Services; Qun Zheng, Georgia Department of Public Health. Youth Risk Behavior Survey (YRBS) state collaborators: Amberlee Baxa, Nevada Division of Public and Behavioral Health; Fred N. Breukelman, Delaware Division of Public Health; Stephanie Bunge, Kentucky Department of Education; Thad Burk, Oklahoma Department of Health; Tara Cooper, Rhode Island Department of Health; Kathleen Courtney, Arkansas Department of Education; Ellen Essick, North Carolina Department of Public Instruction; Robert M. Fiedler, Department of Health \& Mental Hygiene; Jessica H. Gerdes, Illinois State Board of Education; Tori Havins, Arizona Department of Education; Robert Hesia, Hawaii Department of Education; Julane Hill, Nebraska Department of Education; Celeste Jorge, Connecticut Department of Public Health; Sarah Khalidi, Alabama Department of Public Health; Cris Kimbrough, New Mexico Public Education Department; Irene Koffink, New Hampshire Department of Education; Robyn L. Matthews, Indiana Department of Health; Chiniqua N. Milligan, Massachusetts Department of Elementary \& Secondary Education; Sabrina B. Moore, South Carolina Department of Education; LaTina Morgan, Mississippi Department of Education; Martha R. Morrissey, New York Education Department; Kristen Murray, Vermont Department of Health; Birgit A. Shanholtzer, West Virginia Department of Education; Nicholas T. Slotterback, Pennsylvania Department of Education; Daniela E. Torres, California Department of Education; Thomas Troelstrup, Florida Department of Health; Janet S. Wilson, Missouri Department of Health and Senior Services; Jean Zimmerman, Maine Department of Education.

\footnotetext{
${ }^{1}$ Division of Reproductive Health, National Center for Chronic Disease Prevention and Health Promotion, CDC; ${ }^{2}$ Division of Congenital and Developmental Disorders, National Center on Birth Defects and Developmental Disabilities, CDC; ${ }^{3}$ Oak Ridge Institute for Science and Education; ${ }^{4}$ Division of Global Health Protection, Center for Global Health, CDC.
}

Corresponding author: Charlan Kroelinger, ckroelinger@cdc.gov, 770-488-6545.

\section{References}

1. Rasmussen SA, Jamieson DJ, Honein MA, Petersen LR. Zika virus and birth defects-reviewing the evidence for causality. N Engl J Med 2016;374:1981-7. http://dx.doi.org/10.1056/NEJMsr1604338

2. Brasil P, Pereira JP Jr, Raja Gabaglia C, et al. Zika virus infection in pregnant women in Rio de Janeiro-preliminary report. N Engl J Med 2016. Epub March 4, 2016. http://dx.doi.org/10.1056/NEJMoa1602412

3. CDC. Estimated range of Aedes albopictus and Aedes aegypti in the United States, 2016. Atlanta, GA: US Department of Health and Human Services, CDC; 2016. http://www.cdc.gov/zika/pdfs/zika-mosquito-maps.pdf

4. Trussell J. Contraceptive failure in the United States. Contraception 2011;83:397-404. http://dx.doi.org/10.1016/j.contraception.2011.01.021

5. CDC. Zika virus. Atlanta, GA: US Department of Health and Human Services, CDC; 2016. http://www.cdc.gov/zika/index.html

6. Finer LB, Zolna MR. Declines in unintended pregnancy in the United States, 2008-2011. N Engl J Med 2016;374:843-52. http://dx.doi. org/10.1056/NEJMsa1506575

7. Kost K. Unintended pregnancy rates at the state level: estimates for 2010 and trends since 2002. New York, NY: Guttmacher Institute; 2015. https:// www.guttmacher.org/sites/default/files/pdfs/pubs/StateUP2010.pdf

8. Curtis KM, Tepper NK, Jatlaoui TC, et al. U.S. medical eligibility criteria for contraceptive use, 2016. MMWR Recomm Rep 2016;65(No. RR-3).

9. Daniels K, Daugherty J, Jones J, Mosher W. Current contraceptive use and variation by selected characteristics among women aged 15-44: United States, 2011-2013. Natl Health Stat Report 2015;10:1-14.

10. Parks C, Peipert JF. Eliminating health disparities in unintended pregnancy with long-acting reversible contraception (LARC). Am J Obstet Gynecol 2016;214:681-8. http://dx.doi.org/10.1016/j.ajog.2016.02.017

11. Frost JJ, Zolna MR. Contraceptive needs and services, 2013 update. New York, NY: Guttmacher Institute, 2015, https://www.guttmacher. org/pubs/win/contraceptive-needs-2013.pdf

12. Bearak JM, Finer LB, Jerman J, Kavanaugh ML. Changes in out-of-pocket costs for hormonal IUDs after implementation of the Affordable Care Act: an analysis of insurance benefit inquiries. Contraception 2016;93:139-44. http://dx.doi.org/10.1016/j.contraception.2015.08.018

13. Fox J, Barfield W. Decreasing unintended pregnancy: opportunities created by the Affordable Care Act. JAMA 2016. Epub July 25, 2016. http://dx.doi.org/10.1001/jama.2016.8800

14. Kumar N, Brown JD. Access barriers to long acting reversible contraceptives for adolescents. J Adolesc Health 2016. Epub May 28, 2016. http://dx.doi.org/10.1016/j.jadohealth.2016.03.039

15. CDC. Draft interim CDC Zika response plan (CONUS and Hawaii): initial response to Zika virus. Atlanta, GA: US Department of Health and Human Services, CDC; 2016. http://www.cdc.gov/zika/pdfs/zikadraft-interim-conus-plan.pdf 